\title{
Subcutaneous Sarcoidosis in a Nigerian female
}

Yakubu A.A., FWACS, Edino S.T., FWACS, * Mohammed AZ Departments of Surgery and * Pathology, Bayero University Kano Correspondence to: Dr Yakubu A. Ahmed, FWACS, Department of Surgery, Aminu Kano Teaching Hospital, Kano, PMB 3452, Kano E-mail: yakubuaa@yahoo.co.uk Phone +234 8037015339

\section{Summary}

Sarcoidosis is relatively uncommon in indigenous black Africans, especially along the West African coast. There has not been any report of isolated cutaneous sarcoidosis from our center.

We present a patient who was reviewed with an isolated ganglion-like subcutaneous swelling on the ankle region. Excision biopsy revealed a non caseating granulomatous lesion. Systemic evaluation was not remarkable and the lesion was negative for tuberculosis and fungi. The operative wound healed with the use of of a combination therapy with oral chloroquine, prednisolone, and methotrexate. We conclude that cutenous sarcoidosis can occur in the absence of systemic disease and definitive diagnosis is by histological finding of a non caseating granuloma, when other potential causes such as tuberculosis are excluded.

\section{Introduction}

Sarcoidosis is a chronic multisystem disorder of unknown etiology characterized by formation of non caseating epitheloid granulomas and derangement of normal tissue architecture $(1,2)$. It can affect all organs of the body but intrathoracic involvement (pulmonary and hilar nodes) occurs in about $90 \%$ of cases (3). It is a world wide disease with regional variation in incidence, affecting both genders with slight female preponderance (4). All age groups are affected with bimodal peaks between 25-35 years and 45-65 years (4). In the West, the disease is quite common in persons of black descent, the Scandinavians, and the Irish. African Americans (especially women) are more severely affected than whites (5). Sarcoidosis is said to be rare in indigenous Africans, but there are now well validated reports of many clinical cases in Africa $(6,7)$. Africans are thought to present most commonly with extrapulmonary sarcoidosis albeit in late stages. Skin sarcoidosis can arise in isolation or as a component of a systemic disease.

There has not been any report on sarcoidosis from our center. This case is being reported to highlight this unusual skin presentation.

\section{Case Report}

A 65 year old married woman presented with a swelling on the anterior surface of her right ankle joint of six months duration. The swelling was initially small and painless but later increased in size and became painful. There were no other swellings elsewhere, neither was there any preceeding history of trauma. Review of other systems was normal. The lesion was about $4 \times 6 \mathrm{~cm}$ in size, non tender and not fixed to underlying tissues. The overlying skin was grossly normal. A preliminary diagnosis of ganglion of the flexor tendons was made. The complete blood count, urinalysis, serum electrolytes and urea were within normal limits. Surgical excision of the lesion under local anaesthesia revealed a cream white subcutaneous mass not attached to the tendons. The wound was closed primarily. Histology revealed a non caseating granulomatous lesion (fig1). Mantoux test was negative, and the chest radiograph was normal. Acid fast stain and Periodic acid Schiff stain for fungal granuloma were negative.

The incision site failed to heal. Progression of lesion was evident between the gaping edges of the wound. The patient was subsequently placed on prednisolone $40 \mathrm{mg}$ twice daily, oral chloroquine $200 \mathrm{mg}$ daily and intravenous methotrexate $40 \mathrm{mg}$ monthly. After an appreciable improvement the predisolone was tailed off to $5 \mathrm{mg}$ daily. The medications were administered for four months by which time the wound had healed, and no recurrence was noticed at the site.

The patient has been followed up for more than a year 
without any evidence of local recurrence or systemic manifestation.

\section{Discussion}

Infiltration of tissues with noncaseating granuloma is the hallmark of sarcoidosis (1-7). It most commonly affects the lungs, lymph nodes, eyes, bones and skin. Gastrointestinal and neurosarcoidosis, although rare, have been reported. In patients with systemic disease the skin is involved in 20$30 \%$ of cases, while isolated cutaneous sarcoidosis is uncommon occurring in only about $10 \%$ of patients (8); Hence the need to closely evaluate the patients for the probable risk of developing systemic disease at a later date. Such regular evaluation at clinic visits includes chest, ophthalmological, and dermatological examination.

Africans are thought to present most com-

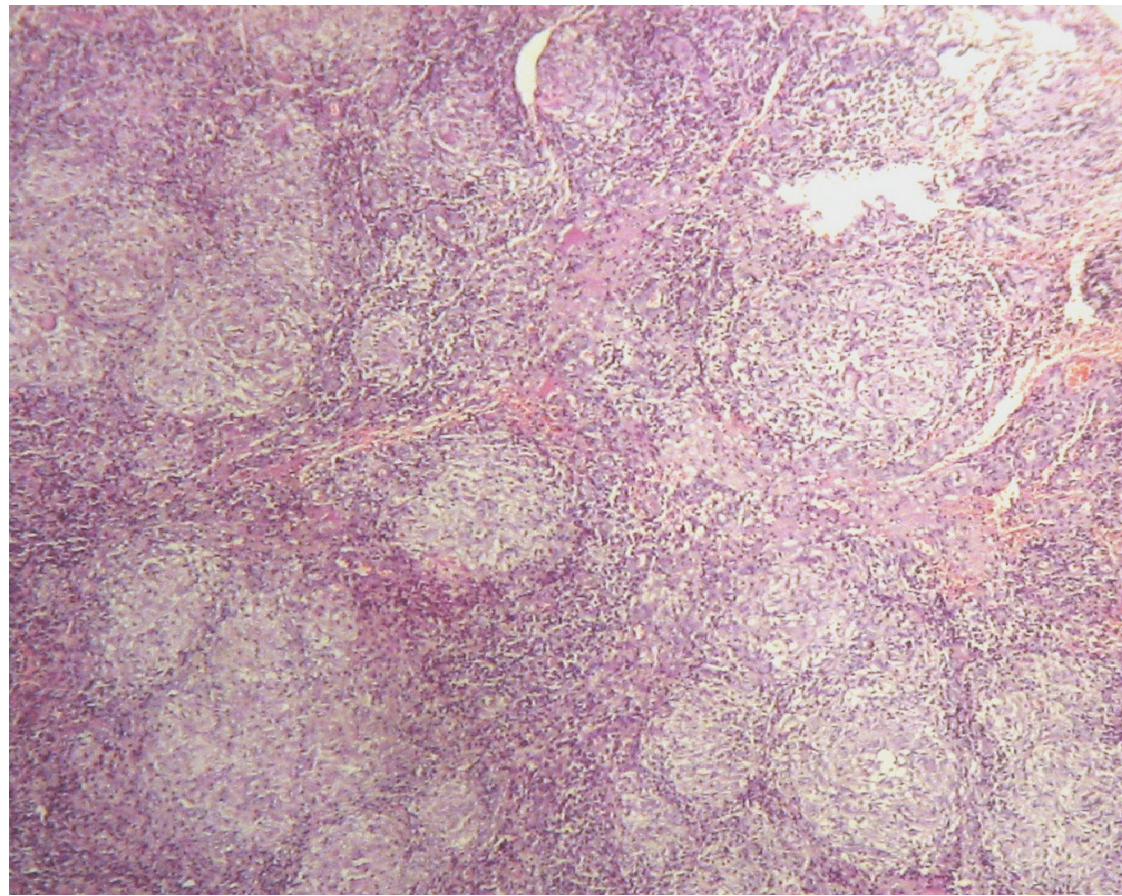

Sarcoidosis $x 4$ showing the granulomas monly with extrapulmonary sarcoidosis with exuberant skin lesions (6). Sarcoid skin lesions are morphologically extremely variable and frequently atypical. They may mimic a number of dermatological conditions (8). They are classified as specific and non specific lesions $(1,2)$. Specific cutaneous lesions include plaques, papules, lupus pernio, scar sarcoid, subcutaneous nodules, ulcers etc., and these lesions exihibit noncaseating granuloma on skin biopsy $(2,9)$. However, in non specific lesions such as erythema nodosum, no granulomas are found (2). Noncaseating granuloma is not pathognomonic of sarcoidosis, as other diseases including mycobacterial, fungal infections, malignancy and berryliosis can also produce the same lesion (1). The diagnosis of sarcoidosis cannot be made with 100\% certainty and no single test can prove the diagnosis. Patients are diagnosed by compatible clinical features and pathological findings and when other potential causes are excluded. The Kveim -Siltzbach test (10) which is specific for sarcoidosis and based on a poorly degradable antigen is lacking in our environment. Intradermal injection of specially prepared tissue from the spleen of a known sarcoidosis patient is biopsied 4 to 6 weeks later. This test is of historical interest only and rarely used in clinical practice as there is no commercially available reagent $(6,7)$.

Although there are many therapeutic options, there are

no clear guidelines based on double blind prospective study for treatment of sarcoidosis, but systemic corticosteroids are the agents of choice in majority of patients. Hydroxychloroquine or chloroquine has been proved to be effective in selected cases of chronic skin sarcoidosis; similarly, cytotoxic agents such as methotrexate, cyclophosphamide, and azathioprine can be used (11). To achieve rapid control of the disease, and reduce the probable complications of prolonged single agent therapy, combination of therapies were used successfully in our patient.

Sarcoidosis can be self limiting or life threatening. The overall prognosis of cutaneous sarcoidosis is primarily dependent on the degree of systemic involvement (1). Mortality is about 5\% and results from pulmonary (progressive pulmonary fibrosis, pulmonary hypertension and right ventricular failure), cardiac (arrhythmias and cardiac failure) and neurologic involvement. We intend to keep following up our patient for any evidence of systemic involvement (10).

In conclusion, a high index of suspicion is necessary for diagnosis of cutaneous sarcoidosis after excluding other causes of noncaseating, non sarcoid lesions. A combination therapy is often helpful in controlling the disease and preventing reccurrence. 


\section{Subcutaneous Sarcoidosis in a Nigerian female}

Yakubu A.A., Edino S.T., Mohammed A.Z.

\section{References}

1. Wilson NJ, King CM. Cutaneous sarcoidosis. Postgrad Med J 1998; 74(877): 649-652

2. Rajani K. Cutaneous sarcoidosis: a dermatologic masquerader. Am Fam Physician 2002; 65:1581-1584.

3. Newman LS, Rose CS, Manier LA. Sarcoidosis. N Engl J Med 1997; 336:1224-1234.

4. Henke CE, Henke LK, Eveheck CM. The epidemiology of sarcoidosi in Rochester MN: a population based study of incidence and survival. Am J Epidemiol 1986; 123: 840-845.

5. Ribicki BA, Meggr M, Popovich J jnr. Racial differences in sarcoidosis incidence: a 5 year study in a health maintenance organization. Am J Epidemiol 1999; 145:234-241.
6. Jacyk WK. Cutaneous sarcoidosis in black South Africans. Int J Dermatol 1999; 38(11):841-5.

7. Alabi GO, George AO. Cutaneous Sarcoidosis and tribal scarification in West Africa. Int J Dermatol 2007; 28(1):29-31.

8. Rajesh M, Sign SN, Bettamishra AB. Cutaneous sarcoidosis without systemic manifestations. Idian J Dermatol 2009; 54:180-182.

9. Gangopadhyay AK. Subcutaneous sarcoidosis: a case report. Indian J Dermatol 1999;44:4.

10. Crystal RG. Sarcoidosis. In: Krasper DL, Braunwald E, Fauci AS et al, eds. Harrison's Principle of Internal Medicine, 16th ed. MacGrawHill Medical Publishing 2005; pp.2017-2023.

11. Bauman RP. Therapeutic options for sarcoidosis: new and old.Curr Opin Pulm Med 2002; 8:464-469. 